\title{
On the Frequency Dependence of the Bias Temperature Instability
}

\author{
T. Grasser*, B. Kaczer ${ }^{\circ}$, H. Reisinger ${ }^{\bullet}$, P.-J. Wagner*, and M. Toledano-Luque ${ }^{\circ}$ \\ ${ }^{*}$ Institute for Microelectronics, TU Wien, Austria $\quad{ }^{\circ}$ imec, Leuven, Belgium $\quad$ Infineon, Munich, Germany
}

\begin{abstract}
An accurate understanding of the duty-factor and frequency dependence of the bias temperature instability (BTI) is essential since it can transfer to significant lifetime benefits. Contradictory reports regarding the frequency dependence have been published in literature, with particularly older studies and those interpreted in the framework of the reaction-diffusion model claiming BTI to be frequency-independent. Newer studies, on the other hand, clearly show a frequency-dependent component of BTI. Here we discuss this frequency dependence using the recently suggested capture/emission time map model, which explains BTI as a collection of a large number of independent first-order reactions of defects with two states. While this model can explain most features observed in DC and low-frequency AC stress experiments, it does not fully capture the experimentally observed frequency and duty-factor dependence. However, when a more accurate defect model is considered, which describes charging of the defect via an intermediate state, good accuracy with experimental data is obtained.
\end{abstract}

\section{INTRODUCTION}

Compared to a conventional DC stress, AC stress patterns can significantly reduce the accumulated degradation [1-4]. In order to properly predict the potentially enormous lifetime benefits, this feature has to be fully understood. For example, assuming that the degradation follows an effective power-law with exponent 0.1 , a reduction of the degradation due to $\mathrm{AC}$ effects by 10,30 , or $50 \%$ results in a lifetime improvement by a factor of 3,35 , and 1000 , respectively.

While all studies report a duty-factor dependence following the ubiquitous step-shaped curve [1,2,5-8], the frequency dependence of BTI appears to be controversial: particularly older studies using slow measurements report frequency independent behavior $[1,9,10]$, while more recent studies have revealed a frequency dependent contribution [3,11-20]. We study the duty-factor and frequency dependence in the light of the recently proposed capture/emission time (CET) map model [7,21-23] and demonstrate that the recoverable component of BTI can only to the first-order be captured by a two-state defect model. By using a three-state defect model, consistent with detailed studies on the microscopic defect properties [24, 25], the experimentally observed frequency dependence can be reproduced for a wide range of technologies.

\section{CAPture/Emission Time Map Model For BTI}

It has recently been shown that both static as well as dynamic BTI can be described by an ensemble of firstorder defect reactions with a wide distribution of capture and emission times [21]. This model has been validated for various combinations of stress and recovery times for NBTI on SiON/pMOSFETs [7] and PBTI on high-k nMOSFETs
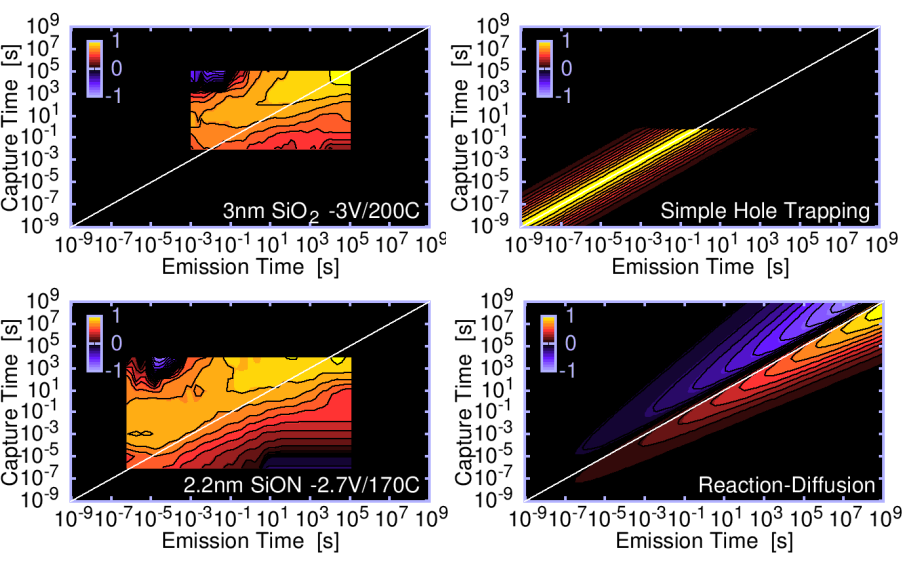

Fig. 1. Left: Experimental CET maps of a $\mathrm{SiO}_{2}$ and a $\mathrm{SiON}$ device directly extracted using eq. (1), which is only possible inside the experimental window. Right: CET maps predicted by the simple hole trapping model and the reactiondiffusion model. Note that the entries in the map above the line $\tau_{\mathrm{e}}=\tau_{\mathrm{c}} / 4$ are negative and do not agree with the experimental maps.

$[7,22]$ both under static and dynamic conditions. The wide distribution of time constants is visualized in the CET map, which can be directly extracted from experimental $\Delta V_{\mathrm{th}}\left(t_{\mathrm{s}}, t_{\mathrm{r}}\right)$ recovery curves for various stress and recovery times $\left(t_{\mathrm{S}}\right.$ and $\left.t_{\mathrm{r}}\right)$ as [21]

$$
g\left(\tau_{\mathrm{c}}, \tau_{\mathrm{e}}\right)=-\frac{\partial^{2} \Delta V_{\mathrm{th}}\left(\tau_{\mathrm{c}}, \tau_{\mathrm{e}}\right)}{\partial \tau_{\mathrm{c}} \partial \tau_{\mathrm{e}}}
$$

The CET map $g\left(\tau_{\mathrm{c}}, \tau_{\mathrm{e}}\right)$ can be visualized as a function of the effective capture and emission times $\tau_{\mathrm{c}}$ and $\tau_{\mathrm{e}}$. In a way, the CET map $g\left(\tau_{\mathrm{c}}, \tau_{\mathrm{e}}\right)$ contains the spectral information of the $\Delta V_{\mathrm{th}}\left(t_{\mathrm{s}}, t_{\mathrm{r}}\right)$ curves, very much like in the Fourier transform theory [7].

The beauty of this empirical but physics-based concept is that the effective CET maps are directly accessible to experiment via eq. (1), thereby clearly demonstrating the wide distribution and weak correlation of the effective capture and emission times. This allows for a convenient evaluation of theoretical models which then have to be able to explain the experimentally observed distribution in $g\left(\tau_{\mathrm{c}}, \tau_{\mathrm{e}}\right)$. Such a comparison is much more intuitive than a comparison in the time-domain. For example, the standard elastic tunneling models predicts a recovery of the form [26,27]

$$
\Delta V_{\mathrm{th}}\left(t_{\mathrm{s}}, t_{\mathrm{r}}\right) \approx A \log \left(1+B t_{\mathrm{r}} / t_{\mathrm{s}}\right)
$$

with parameters $A$ and $B$. At a first glance, this appears compatible with experimental recovery data and similar models have been used frequently [28-30]. However, the CET map predicted by the elastic tunneling model, obtained from eq. (2) 

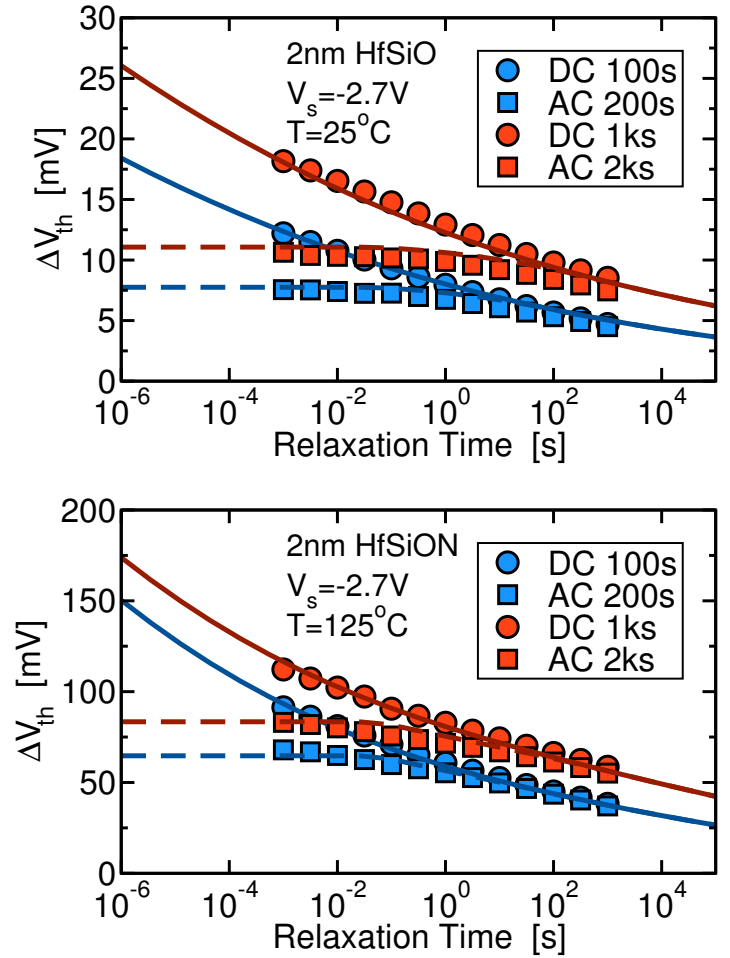

Fig. 2. Two extreme cases of recovery after DC and AC stress for the non-nitrided $\mathrm{HfSiO}$ device at $25^{\circ} \mathrm{C}$ (left) and the nitrided $\mathrm{HfSiON}$ device at $125^{\circ} \mathrm{C}$. As already demonstrated in $[7,22]$ for PBTI, recovery after AC stress of duration $2 \times t_{\mathrm{s}}$ merges with the $\mathrm{DC}$ recovery after stress of duration $t_{\mathrm{S}}$ at $t_{\mathrm{r}}=t_{\mathrm{s}}$. The analytic model (solid lines for DC and dashed lines for AC) reproduces the data very well.

according to eq. (1), is

$$
\tilde{g}\left(\tau_{\mathrm{c}}, \tau_{\mathrm{e}}\right)=\frac{A B}{\left(B+\tau_{\mathrm{e}} / \tau_{\mathrm{c}}\right)^{2}} \frac{\tau_{\mathrm{e}}}{\tau_{\mathrm{c}}} \quad \text { for } \quad \tau_{\mathrm{c}}<t_{\max }
$$

which is a logistic distribution of the ratio $\tau_{\mathrm{e}} / \tau_{\mathrm{c}}$. The maximum emission time $t_{\max }$ is on the order of seconds in thin $(<$ $2 \mathrm{~nm}$ ) oxides. Furthermore, the correlation between $\tau_{\mathrm{e}}$ and $\tau_{\mathrm{c}}$ is much stronger than observed experimentally, see Fig. 1 (top), ruling this model out solely based on the CET map [23,27] (not to mention the wrong bias and temperature dependence). Similar conclusions hold for the reaction-diffusion model [10], which even produces negative entries [27], see Fig. 1 (bottom).

Note, however, that the effective time constants in the CET map do not directly correspond to physical defect parameters like thermodynamic energy-levels or relaxation energies but are functions of them [27]. In this sense we wish to highlight that the CET map model is not a new model meant to replace our previous modeling efforts such as [24,31]. Rather, the CET map model provides a convenient transformation of the experimental data to help understand the wide distribution of time constants. In particular, the CET map model can intuitively explain the effect of stress and recovery on a distributed set of defects, including low-frequency AC effects [21,23].

In order to avoid technical issues with the second-order numerical derivative of experimental data and to facilitate our understanding of the distribution, we have recently suggested an analytic model for the CET map [23]. An additional benefit of this analytical model is that it allows for extrapolation out
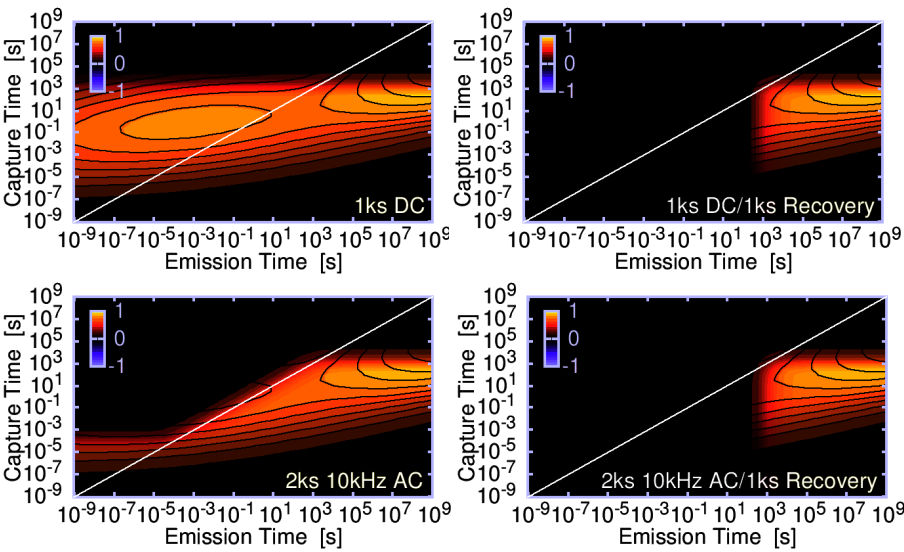

Fig. 3. Left: The occupied defects in the CET map of a HfSiO device following $1 \mathrm{ks}$ of DC stress (top) and $2 \mathrm{ks}$ of $50 \% \mathrm{AC}$ stress (bottom). Right: According to the first-order model, after a recovery time of $t_{\mathrm{r}}=1 \mathrm{ks}$, all defects with $\tau_{\mathrm{e}}<1 \mathrm{ks}$ have been discharged, and the degradation after DC stress (top) equals the one after AC stress (bottom).

of the experimental window, allowing for an improved lifetime prediction. The analytic CET map uses a recoverable $(\mathrm{R})$ and a permanent $(\mathrm{P})$ component, both of which are represented by bivariate normal distributions for the effective activation energies of the correlated capture and emission times. Given recent experimental evidence [21,24,32-34], all time constants are assumed to be thermally activated, $\tau=\tau_{0} \exp \left(\beta E_{\mathrm{A}}\right)$, with $\beta=1 / \mathrm{k}_{\mathrm{B}} T_{\mathrm{L}}, T_{\mathrm{L}}$ the lattice temperature, and $\mathrm{k}_{\mathrm{B}}$ the Boltzmann constant. Based on the analytic CET map, the overall degradation can be analytically calculated. Compact expressions for digital switching between two discrete gate bias levels have been given for any combination of stress time/recovery time/duty factor, with excellent agreement to measurement data in the very wide window of stress and relaxation times from $1 \mu$ s to $100 \mathrm{ks}$ [23]. While the accuracy of the model for DC and low-frequency AC stress is remarkable, see numerous comparisons in [23] and Fig. 2, discrepancies can be observed at higher frequencies, which will be the subject of the present paper.

\section{FREQUENCY DEPENDENCE}

Our discussion is based on the following simple prediction of the model for an AC stress with duty-factor $\alpha$ and thus on/off ratio $\gamma=\alpha /(1-\alpha)$ : Given that the duration of the AC stress equals $t_{\mathrm{S}} / \alpha$, with the cumulative stress time $t_{\mathrm{s}}$, the build-up of slowly recoverable traps with $\tau_{\mathrm{e}}>t_{\mathrm{s}} / \gamma$ is the same under both conditions [7], independent of frequency. The only difference is in the occupancy of the faster traps with $\tau_{\mathrm{e}}<t_{\mathrm{s}} / \gamma$. However, after a recovery time of $t_{\mathrm{M}}=t_{\mathrm{S}} / \gamma$, these differences are wiped out, and AC and DC degradation must be the same. For example, with a duty-factor of $50 \%$, we have $t_{\mathrm{M}}=t_{\mathrm{s}}$. Then, recovery after a $2 \mathrm{ks}$ AC stress must match the recovery of a $1 \mathrm{ks}$ DC stress after $t_{\mathrm{M}}=1 \mathrm{ks}$. This is a simple consequence of the first-order reaction and completely independent of the actual distribution of the capture and emission times. Indeed, the merging of AC and DC recovery at the predicted recovery time has been experimentally confirmed for low-frequency AC PBTI on high-k $[6,7,22]$ and low-frequency AC NBTI on 


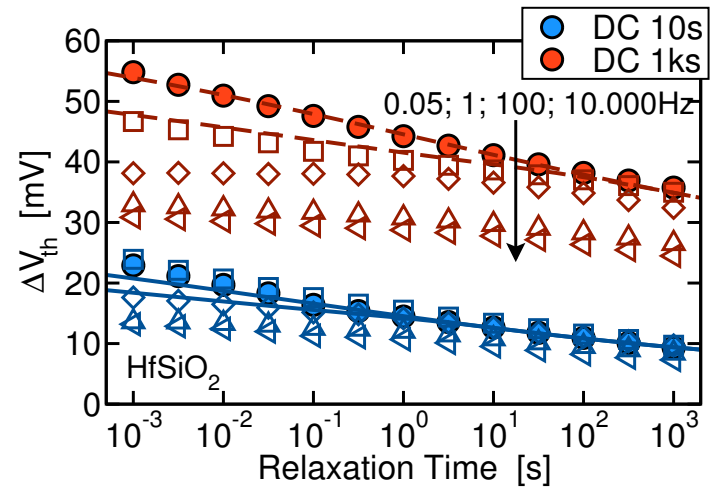

Fig. 4. While at $50 \mathrm{mHz}$ the model (lines) agrees well with the data (syms), the model overestimates the degradation at higher frequencies, particularly in the non-nitrided sample shown above.

$\mathrm{SiO}_{2}, \mathrm{SiON}, \mathrm{HfSiO}$ and $\mathrm{HfSiON}$ samples [23], see Fig. 2. An example demonstrating the occupancies of the defects in the CET map for a HfSiO device both directly after stress and at the merging point $t_{\mathrm{M}}$ is given in Fig. 2. The occupancy times the defect density in the CET map is shown in Fig. 3: while the occupancy is significantly different right after stress (left panels), they are the same at $t_{\mathrm{M}}$ (right panels).

One possible explanation for the controversial reports on the frequency dependence is that during AC stress the degradation oscillates between the cumulative stress and recovery levels. Any fast measurement result will therefore critically depend on the measurement delay which, ideally, should be synchronized with the on/off stress condition. However, according to the first-order model [7,22], any remnants of this frequencydependent component will have recovered after $t_{\mathrm{r}}=t_{\mathrm{M}}$. As such, in order to remove this uncertainty, we propose to check for a frequency dependent contribution to BTI with a deliberately large delay at the merging point $t_{\mathrm{M}}$. This would also be favorable from the perspective of reaction-diffusionbased theories which claim that the frequency-dependent holetrapping component vanishes after a few seconds, leaving behind the frequency-independent $\mathrm{RD}$ contribution [8,35].

We have experimentally tested the validity of the above firstorder model prediction on a number of technologies $\left(\mathrm{SiO}_{2}\right.$, $\mathrm{SiON}, \mathrm{HfSiO}$, and HfSiON) and consistently found that the predicted merging only occurs at low frequencies $(<1 \mathrm{~Hz}-$ $100 \mathrm{~Hz}$ ). In Fig. 4 it is demonstrated how the degradation level decreases with increasing frequency. The discrepancy is about $10 \%$ for the SiON device but can reach levels of $30 \%$ in the HfSiO device measured, see Fig. 5. This effect cannot be reproduced by the first-order model no matter what parameters are used, since it is a direct consequence of the underlying first-order reaction. We conclude that either $\mathbf{R}$ or $\mathbf{P}$ (or both) cannot be described by a first-order reaction.

In order to attempt a separation of $\mathrm{R}$ and $\mathrm{P}$, the $\mathrm{AC}$ stress is performed with different AC-off voltages, since it has been shown that $\mathrm{R}$ recovers much quicker under positive bias [24, 36-39]. Indeed, as shown in Fig. 6, particularly at an AC-off level of $+1 \mathrm{~V}$, no frequency dependence is observed following the merging point $t_{\mathrm{M}}$. We conclude that $\mathrm{P}$ can be described by a first-order model while $\mathrm{R}$ requires additional measures.

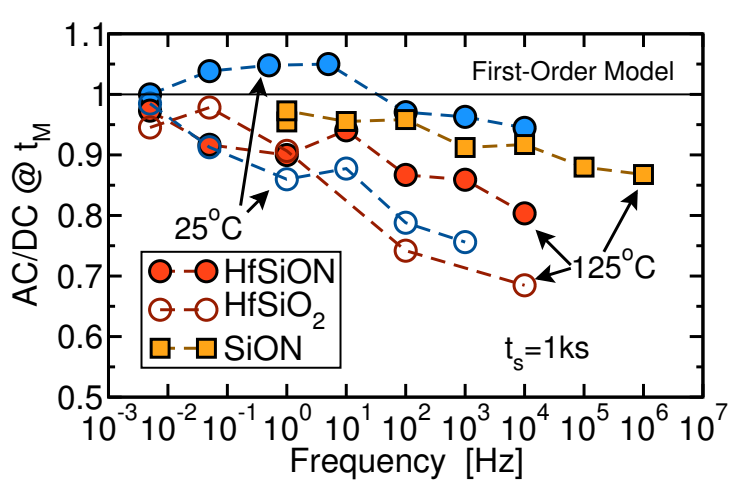

Fig. 5. Ratio of the $\mathrm{AC}(50 \%, 2 \mathrm{ks})$ vs. $\mathrm{DC}(1 \mathrm{ks})$ recovery at the theoretical merging point $t_{\mathrm{M}}=1 \mathrm{ks}$. At higher frequencies, the recovering $\mathrm{AC}$ degradation does no longer merge at $t_{\mathrm{M}}$. Shown is data (symbols) for a HfSiO device, where the effect is relatively strong, a HfSiON, and a SiON device. According to the first-order model, this ratio should be close to unity, which is obviously not the case.

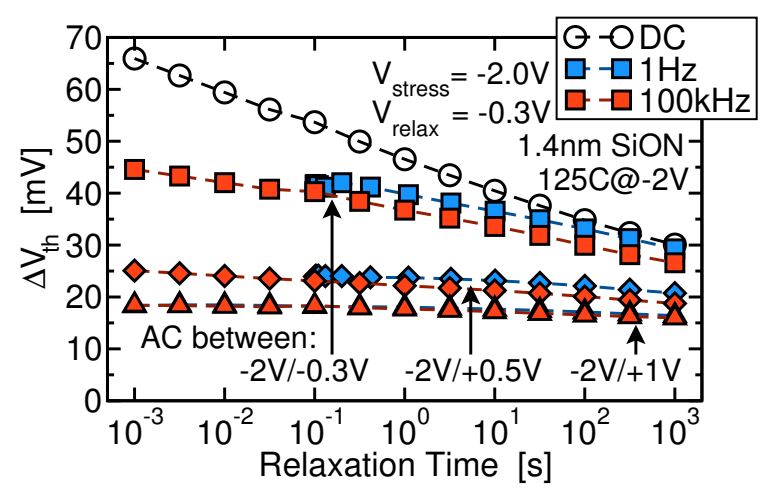

Fig. 6. When an AC stress is performed using the same on/off voltages as used during the subsequent recovery, the AC recovery after $2 \mathrm{ks}$ stress merges with the DC recovery after $1 \mathrm{ks}$ stress when $t_{\mathrm{r}}=1 \mathrm{ks}$, provided the frequency is low enough. When a more positive AC-off level is used instead of $V_{\mathrm{r}}=-0.3 \mathrm{~V}$, the frequency dependence gradually begins to disappear. This is strong evidence that $\mathrm{R}$ is frequency dependent but $\mathrm{P}$ is not [28].

\section{AdVAnced CET Map Model}

The CET map model is based on the assumption that the multi-state defect behavior observed previously [24] can be represented by an effective first-order (two-state) model. In particular, it was observed that the bias dependence of the effective capture time constant could be best explained by treating the change in the charge-state and the subsequent structural relaxation as a two-step process via an intermediate state $2^{\prime}$. The initial precursor state is given by 1 and the positively charged state is given by 2 . Such a three-state model has also been used to explain the frequency dependence of PBTI in high-k pMOSFETs [16] under the assumption that the defects are negative- $U$ centers. In addition it was observed that recovery may proceed via a metastable neutral state $1^{\prime}$, which is also responsible for the switching trap behavior [31]. This pathway can dramatically reduce the emission time constant when the gate voltage goes below $V_{\text {th }}$ [40]. The full transition state diagram is shown below: 


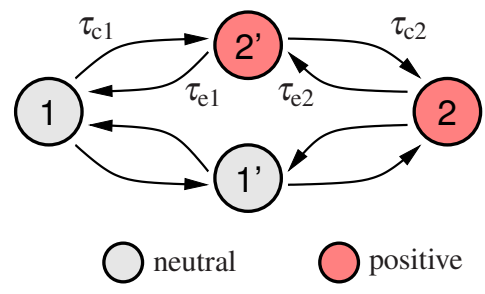

In order to get a first impression of the behavior of a two-step charging behavior, we will restrict our modeling attempts to the case $\left|V_{\mathrm{G}}\right|>\left|V_{\mathrm{th}}\right|$ and neglect the metastable neutral state $1^{\prime}$. As such, we obtain the following simplified defect model

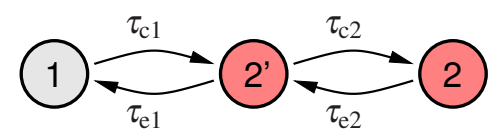

where the time constants $\tau_{\mathrm{c} 1}$ and $\tau_{\mathrm{e} 1}$ connect the metastable state with the neutral precursor state while $\tau_{\mathrm{c} 2}$ and $\tau_{\mathrm{e} 2}$ connect the metastable state with the positively charged state. Note that according to our previous model, only $\tau_{\mathrm{c} 1}$ and $\tau_{\mathrm{e} 1}$ depend on $V_{\mathrm{G}}$, while $\tau_{\mathrm{c} 2}$ and $\tau_{\mathrm{e} 2}$ are thermal transitions over a barrier. Also, $\tau_{\mathrm{e} 1}\left(V_{\mathrm{th}}\right)$ is very large and we neglect the contribution of state $2^{\prime}$ to $\Delta V_{\text {th }}$.

Under DC conditions, the three-state defect can be approximated by a first-order two-state model using the effective capture and emission time constants [27]

$$
\begin{aligned}
& \tau_{\mathrm{c}}=\tau_{\mathrm{c} 1}+\tau_{\mathrm{c} 2}\left(1+\tau_{\mathrm{c} 1} / \tau_{\mathrm{e} 1}\right), \\
& \tau_{\mathrm{e}}=\tau_{\mathrm{e} 1}+\tau_{\mathrm{e} 2}\left(1+\tau_{\mathrm{e} 1} / \tau_{\mathrm{c} 2}\right) .
\end{aligned}
$$

The approximation of a three-state defect by an effective twostate defect is valid provided the frequency does not become too large, in particular, $f<1 / \tau_{\mathrm{c} 1}$.

For higher frequencies, the metastable state $2^{\prime}$ acts like a low-pass filter. In order to further simplify the model, we only consider the forward rates during stress

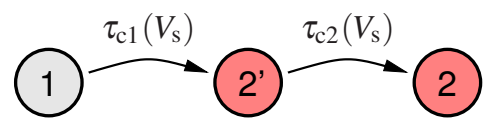

and only the backward rates during recovery

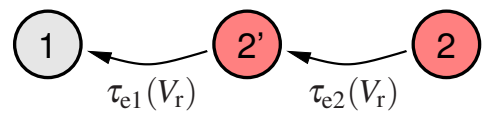

where $V_{\mathrm{s}}$ and $V_{\mathrm{r}}$ are the gate voltages during stress and recovery, respectively. The low-pass effect is shown in Fig. 7: while the transition from state 1 to $2^{\prime}$ shows the behavior of a two-state defect, that is, has a frequency dependence of the plateau value but not of the time constant, the transition from state $2^{\prime}$ to 2 shows a frequency-dependent effective capture time.

As can be seen in that simple example, the effective capture time constant can show a marked frequency dependence in the three-state model. Such a frequency dependence is only obtained when $\tau_{\mathrm{c} 1} \gg \tau_{\mathrm{e} 1}$ and $f<1 / \tau_{\mathrm{c} 1}$, meaning that state $2^{\prime}$ is completely emptied in every cycle. Otherwise, no frequency dependence is obtained. As such, for low enough frequencies,

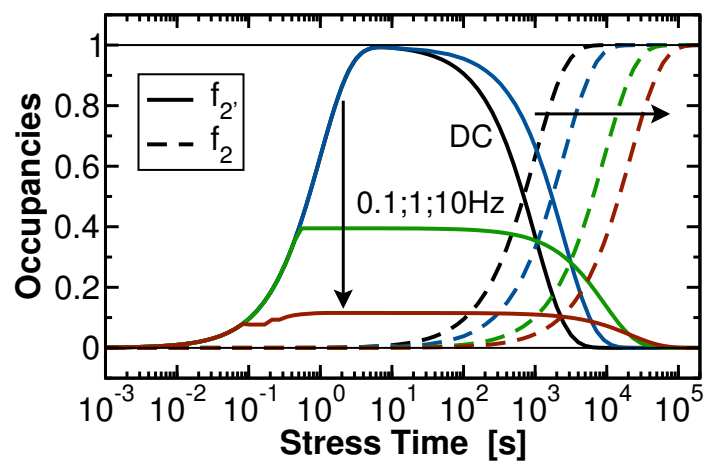

Fig. 7. Based on the detailed microscopic study of individual defects [24], we now describe charging/discharging of state 2 via a metastable state $2^{\prime}$. Initially, state $2^{\prime}$ is charged. When $\tau_{\mathrm{c} 1} \gg \tau_{\mathrm{e} 1}$ and larger than the inverse frequency, state $2^{\prime}$ is completely discharged in every cycle, inhibiting the charging of state 2 . In contrast, in a two-state model only the final occupancy level depends on frequency but not the effective capture time [7,33].
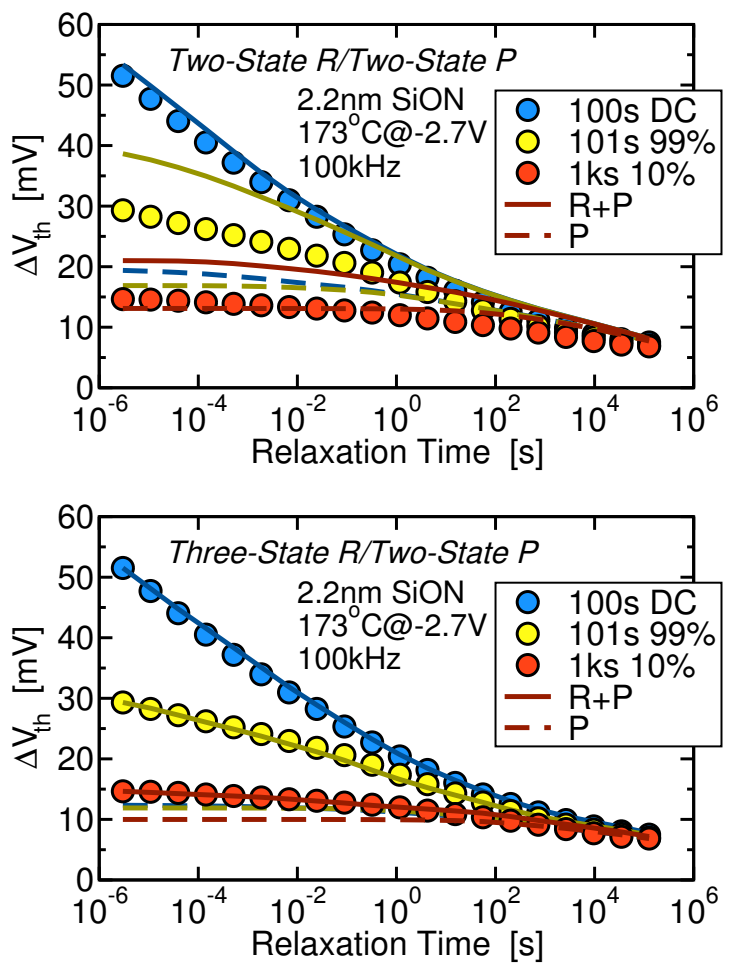

Fig. 8. Top: The experimental recovery curves merge much later than predicted by the first-order model. Also, the degradation predicted by the firstorder model is nearly $25 \%$ too large. Bottom: By using frequency-dependent three-state defects for $\mathrm{R}$, the experimental behavior can be well reproduced.

the three-state model simplifies to the two-state model. In particular, this implies that the predictions of the DC and lowfrequency behavior are not affected by this extension of the model, provided the stress and relaxation times are larger than $\tau_{\mathrm{c} 1}$ and $\tau_{\mathrm{e} 1}$. As with the two-state model $[7,33]$, the response of the three-state defect to an AC signal can be evaluated in closed form, without the tedious requirement to resolve millions of cycles. While the derivation of the expressions is rather cumbersome compared to the elegant and simple solution of the two-state defect, they lead to a very simple results. In essence, under $\mathrm{AC}$ conditions, the capture time 

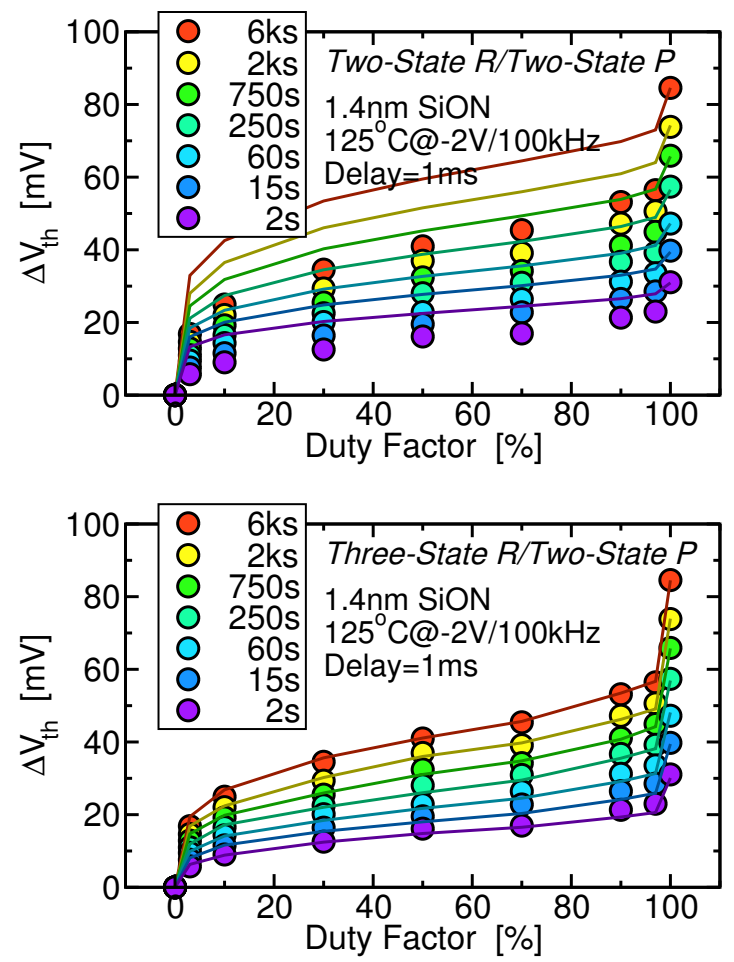

Fig. 9. Top: The first-order model (lines) can only qualitatively reproduce the experimental duty-factor dependence (symbols). Data was recorded for increasing stress-times and measured with a delay of $1 \mathrm{~ms}$. Bottom: By using three-state defects for $\mathrm{R}$, the duty-factor dependence can be accurately reproduced (lines).

constant $\tau_{\mathrm{c} 2}$ of a three-state defect has to be replaced by

$$
\tau_{\mathrm{c} 2} \rightarrow \tau_{\mathrm{c} 2} / w
$$

The factor $w$ introduces a frequency dependence and is smaller than unity. Approximately, we have

$$
w \approx \begin{cases}1 & \text { for } f<f_{\mathrm{c}} \\ \alpha / 2 \tau_{\mathrm{c} 1} f & \text { for } f_{\mathrm{c}}<f<f_{\mathrm{m}} \\ \tau_{\mathrm{e} 1} \gamma / \tau_{\mathrm{c} 1} & \text { for } f_{\mathrm{m}}<f\end{cases}
$$

with the corner frequency $f_{\mathrm{c}}=\alpha / 2 \tau_{\mathrm{c} 1}$ and the maximum frequency $f_{\mathrm{m}}=(1-\alpha) / 2 \tau_{\mathrm{e} 1}$. Details of the derivation and additional consequences of the model will be published elsewhere. Using the above substitution, the analytical formulas given previously [23] remain valid, allowing for a very efficient analytic calculation of the AC degradation as a function of arbitrary stress and recovery times. Typical values used for $\tau_{\mathrm{c} 1}$ are about $1 \mathrm{~ms}$ and $\tau_{\mathrm{e} 1} \approx 0 \mathrm{~s}$.

First, the model is evaluated on experimental data designed to reveal the merging point $t_{\mathrm{M}}$ using 3 different duty-factors, see Fig. 8. As observed previously [7], the prediction of the two-state model is about $25 \%$ higher than the experimental data while the three-state model can capture this effect.

Second, although the two-state model can explain the qualitative features of the duty-factor dependence, comparison to experimental data remains unsatisfactory, see Fig. 9 (top). In particular, it is impossible to introduce a large difference between $\alpha=100 \%$ (DC) and $\alpha=50 \%$ (AC) without signifi-
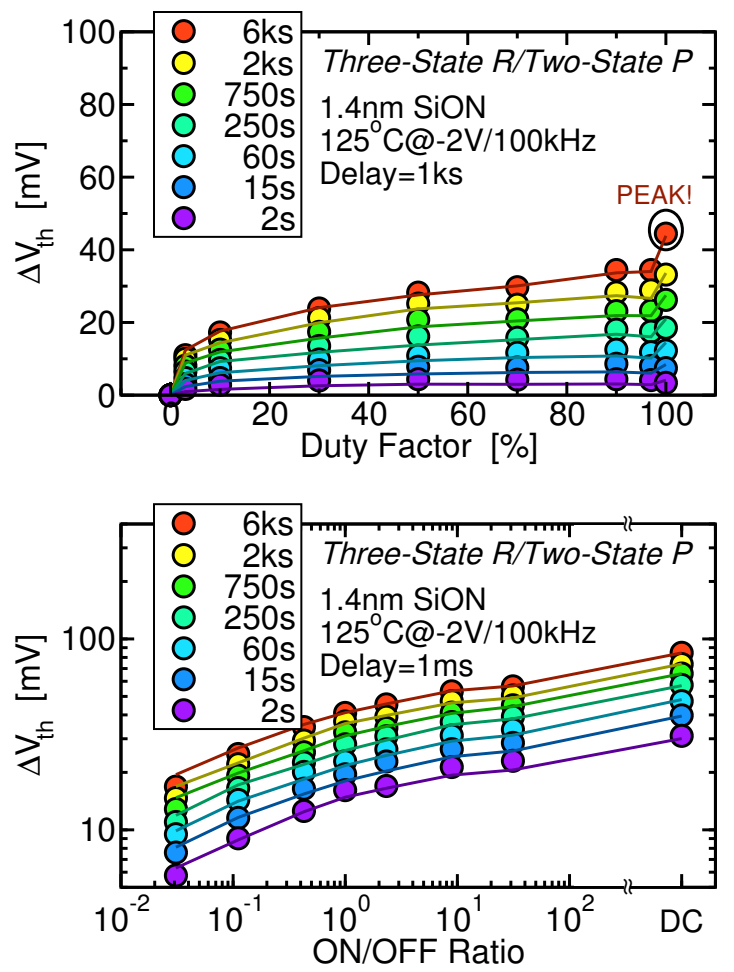

Fig. 10. Top: Even after a measurement delay of $1 \mathrm{ks}$, the ubiquitous shape of the duty-factor dependence is preserved, particularly the striking peak for $\mathrm{DF} \rightarrow 100 \%$. Bottom: Same comparison of data and simulation as in Fig. 9 (bottom) plotted versus the on/off ratio $\gamma=\alpha /(1-\alpha)$, which more clearly displays the data for small and large values of $\gamma$. [7].

cantly impacting the model accuracy for DC stress. As such, the simulated step is never as pronounced as that observed experimentally. Much better agreement is obtained with the three-state model as shown in Fig. 9 (bottom) and Fig. 10. Remarkably, the ubiquitous shape of the curve is preserved even after a recovery time of $1 \mathrm{ks}$, including the peak at $\alpha=100 \%$. This is in stark contrast to RD theory papers [35], which claim that this peak is an artifact due to hole trapping and that the 'real' duty-factor dependence is given by RD theory. Quite to the contrary, given the preserved peak, we conclude again that hole trapping essentially determines NBTI even for longer stress and recovery times [8].

Third, the model is evaluated against the data recorded on the HfSiO device, which shows the strongest frequency dependence of $30 \%$ at $10 \mathrm{kHz}$. Again, while the two-state model shows no frequency dependence after a sufficiently long recovery period, the three-state model is consistent with the experimental data as shown in Fig. 11. In particular, even after a long recovery period, AC and DC data do not merge.

Finally, we evaluate the bias dependence of the three-state CET map model to validate the assumptions derived from Fig. 6. For this, the parameters of the CET map need to be made bias-dependent. It has previously been shown that a considerable fraction of the BTI defects are switching traps, that is, contain at least three states [31]. These switching traps show an exponential dependence on the recovery bias below the threshold voltage [24]. Thus, to the first order, we capture the effect of the recovery voltage on the CET map by 

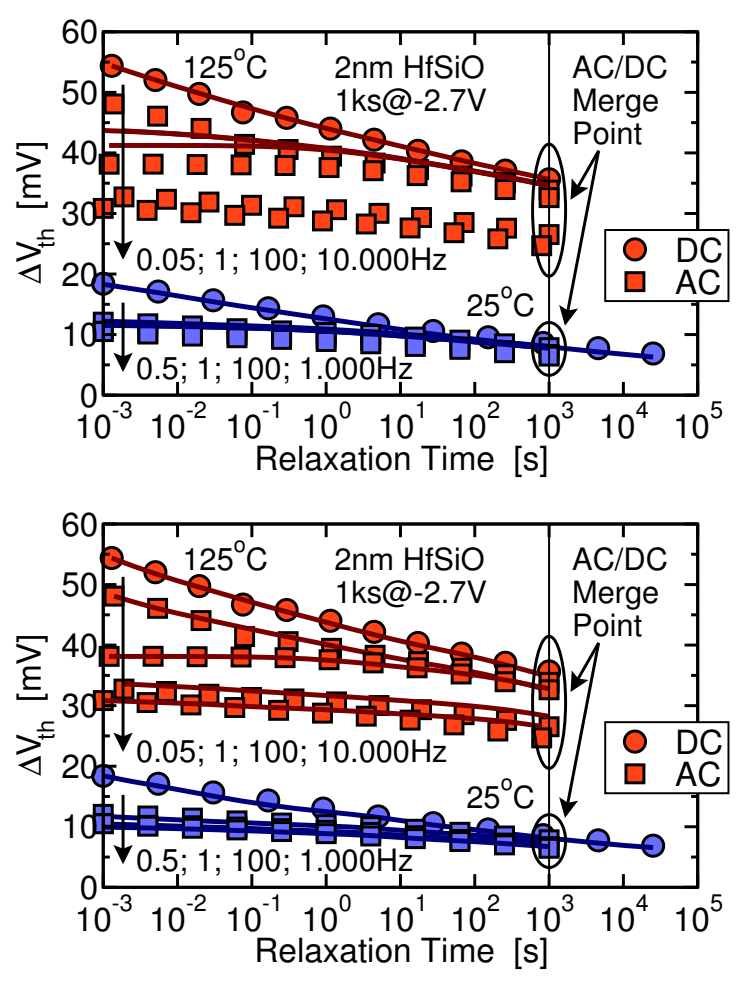

Fig. 11. Top: Simulated (lines) vs. experimental (symbols) recovery after DC and AC stress for the HfSiO device at two temperatures. The merging point predicted by the simple first-order model is experimentally only observed at low frequencies. Bottom: The discrepancy can be removed by assuming a three-state R component (lines).

making the mean emission time of $\mathrm{R}$ bias-dependent. In fact, a simple linear relationship which changed the mean value of the effective activation energy by $300 \mathrm{mV}$ for a change by $1 \mathrm{~V}$ in the gate bias proved to be sufficient. Figs. 12 and 13 shows the evaluation of this simple model against experimental data using 4 different AC-off voltages. Again, the three-state model captures the essence.

\section{Conclusions}

We have studied the frequency dependence of BTI by using our previously developed capture/emission time map model, which is based on first-order reactions. While previous studies on the frequency dependence published in literature probably suffered from various issues - such as unspecified delay and a mixture of $\mathrm{AC}$ and recovery effects in the measured degradation level - our study deliberately uses long recovery times equal to the theoretical first-order AC/DC merging point. The observed deviation from the first-order model means that defect charging follows first-order kinetics only in the low-frequency limit. Consistent with the previously described switching traps, introduction of a third defect state provides a good description of the experimentally observed frequency dependence for a number of different technologies.

\section{ACKNOWLEDGMENTS}

The research leading to these results has received funding from the FWF project $\mathrm{n}^{\circ} 23390-\mathrm{M} 24$ and the European Community's FP7 project $\mathrm{n}^{\circ} 261868$ (MORDRED)
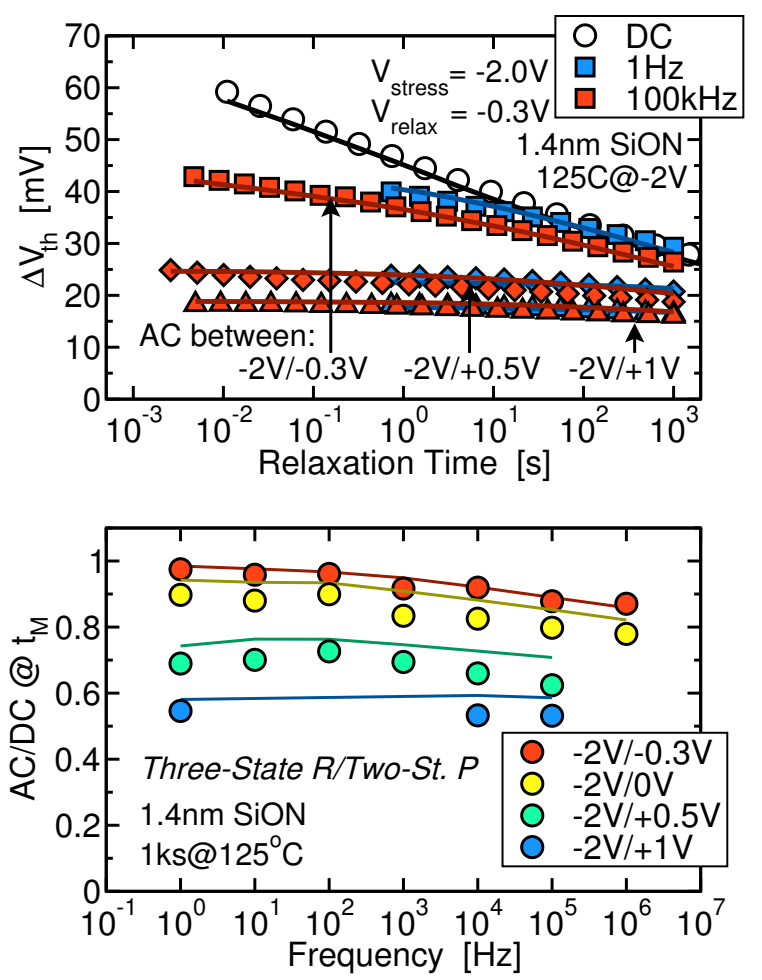

Fig. 12. The experimental behavior at different AC-off voltages (Fig. 6) can be explained by simply shifting the mean value of the effective emission activation energy associated with $\mathrm{R}$ by $300 \mathrm{mV} / \mathrm{V}$. Top: Comparison of model (lines) to data (symbols) for some selected recovery traces. Bottom: Comparison of the ratio $\mathrm{AC}\left(2 \times t_{\mathrm{s}}\right) / \mathrm{DC}\left(t_{\mathrm{s}}\right)$ at $t_{\mathrm{r}}=t_{\mathrm{M}}$ using four different ACoff voltages. The simple model (lines) nicely reproduces the main features of the data (symbols).
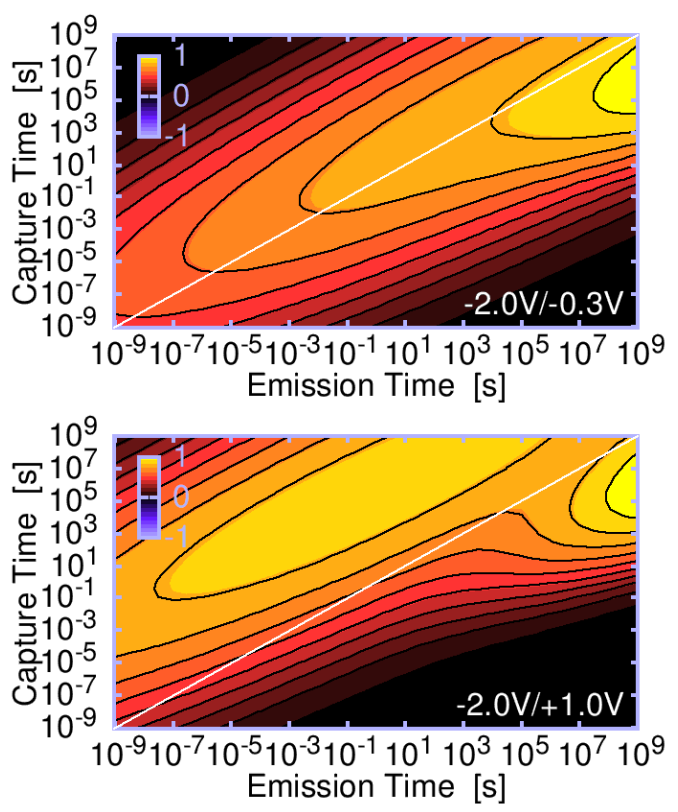

Fig. 13. Top: The CET map belonging to Fig. 12 for the AC-on/off levels of $-2 \mathrm{~V}$ and $-0.3 \mathrm{~V}$ corresponding to the data. Bottom: The empirically modified CET map for the AC-on/off levels of $-2 \mathrm{~V}$ and $+1 \mathrm{~V}$ is obtained by simply shifting the mean effective emission activation energy of $R$ by $\approx 400 \mathrm{mV}$. This results in a significant shift of the whole $\mathrm{R}$ distribution with respect to $\mathrm{P}$, the latter then determining the relaxation behavior. 


\section{REFERENCES}

[1] R. Fernández, B. Kaczer, A. Nackaerts, S. Demuynck, R. Rodriguez, M. Nafria, and G. Groeseneken, "AC NBTI Studied in the $1 \mathrm{~Hz}-2 \mathrm{GHz}$ Range on Dedicated On-Chip CMOS Circuits," in Proc. Intl.Electron Devices Meeting (IEDM), 2006, pp. 337-340.

[2] T. Grasser, B. Kaczer, P. Hehenberger, W. Goes, R. O'Connor, H. Reisinger, W. Gustin, and C. Schlünder, "Simultaneous Extraction of Recoverable and Permanent Components Contributing to BiasTemperature Instability," in Proc. Intl.Electron Devices Meeting (IEDM), 2007, pp. 801-804.

[3] T. Nigam, "Pulse-Stress Dependence of NBTI Degradation and Its Impact on Circuits," IEEE Trans.Dev.Mat.Rel., vol. 8, no. 1, pp. 72$78,2008$.

[4] Z. Teo, A. Boo, D. Ang, and K. Leong, "On the Cyclic Threshold Voltage Shift of Dynamic Negative-Bias Temperature Instability," in Proc. Intl.Rel.Phys.Symp. (IRPS), 2011, pp. 943-947.

[5] B. Kaczer, T. Grasser, P. Roussel, J. Martin-Martinez, R. O'Connor, B. O'Sullivan, and G. Groeseneken, "Ubiquitous Relaxation in BTI Stressing-New Evaluation and Insights," in Proc. Intl.Rel.Phys.Symp. (IRPS), 2008, pp. 20-27.

[6] K. Zhao, J. Stathis, A. Kerber, and E. Cartier, "PBTI Relaxation Dynamics after AC vs. DC Stress in High-k/Metal Gate Stacks," in Proc. Intl.Rel.Phys.Symp. (IRPS), Apr. 2010, pp. 50-54.

[7] H. Reisinger, T. Grasser, K. Ermisch, H. Nielen, W. Gustin, and C. Schlünder, "Understanding and Modeling AC BTI," in Proc. Intl.Rel.Phys.Symp. (IRPS), Apr. 2011, pp. 597-604.

[8] T. Grasser, B. Kaczer, W. Goes, H. Reisinger, T. Aichinger, P. Hehenberger, P.-J. Wagner, F. Schanovsky, J. Franco, M. Toledano-Luque, and M. Nelhiebel, "The Paradigm Shift in Understanding the Bias Temperature Instability: From Reaction-Diffusion to Switching Oxide Traps," IEEE Trans.Electron Devices, vol. 58, no. 11, pp. 3652-3666, 2011.

[9] G. Chen, M. Li, C. Ang, J. Zheng, and D. Kwong, "Dynamic NBTI of p-MOS Transistors and its Impact on MOSFET Scaling," IEEE Electron Device Lett., vol. 23, no. 12, pp. 734 - 736, dec 2002.

[10] M. Alam, "A Critical Examination of the Mechanics of Dynamic NBTI for pMOSFETs," in Proc. Intl.Electron Devices Meeting (IEDM), 2003, pp. 345-348.

[11] W. Abadeer and W. Ellis, "Behavior of NBTI under AC Dynamic Circuit Conditions," in Proc. Intl.Rel.Phys.Symp. (IRPS), 2003, pp. 17-22.

[12] V. Huard, M. Denais, F. Perrier, and C. Parthasarathy, "Static and Dynamic NBTI Stress in pMOS Transistors," in Proc. Insulating Films Semicond. (INFOS), 2003, pp. 1-2.

[13] S. Chakravarthi, A. Krishnan, V. Reddy, C. Machala, and S. Krishnan, "A Comprehensive Framework for Predictive Modeling of Negative Bias Temperature Instability," in Proc. Intl.Rel.Phys.Symp. (IRPS), 2004, pp. 273-282.

[14] S. Mahapatra, M. Alam, P. Kumar, T. Dalei, and D. Saha, "Mechanism of Negative Bias Temperature Instability in CMOS Devices: Degradation, Recovery and Impact of Nitrogen," in Proc. Intl.Electron Devices Meeting (IEDM), 2004, pp. 105-108.

[15] V. Huard, M. Denais, F. Perrier, N. Revil, C. Parthasarathy, A. Bravaix, and E. Vincent, "A Thorough Investigation of MOSFETs NBTI Degradation," Microelectronics Reliability, vol. 45, no. 1, pp. 83-98, 2005.

[16] T. Yang, C. Shen, M.-F. Li, C. Ang, C. Zhu, Y.-C. Yeo, G. Samudra, S. Rustagi, M. Yu, and D.-L. Kwong, "Fast DNBTI Components in pMOSFET with SiON Dielectric," IEEE Electron Device Lett., vol. 26, no. 11, pp. 826-828, 2005.

[17] A. Krishnan, C. Chancellor, S. Chakravarthi, P. Nicollian, V. Reddy, A. Varghese, R. Khamankar, and S. Krishnan, "Material Dependence of Hydrogen Diffusion: Implications for NBTI Degradation," in Proc. Intl.Electron Devices Meeting (IEDM), 2005, pp. 688-691.

[18] S. Zhu, A. Nakajima, T. Ohashi, and H. Miyake, "Interface Trap Generation Induced by Charge Pumping Current Under Dynamic Oxide Field Stresses," IEEE Electron Device Lett., vol. 26, no. 3, pp. 216-219, 2005.

[19] Y. Mitani, H. Satake, and A. Toriumi, "Influence of Nitrogen on Negative Bias Temperature Instability in Ultrathin SiON," IEEE Trans.Dev.Mat.Rel., vol. 8, no. 1, pp. 6-13, 2008.

[20] S. Wang, D. Ang, and G. Du, "Effect of Nitrogen on the Frequency Dependence of Dynamic NBTI-Induced Threshold-Voltage Shift of the Ultrathin Oxynitride Gate P-MOSFET," IEEE Electron Device Lett., vol. 29 , no. 5 , pp. $483-486,2008$.
[21] H. Reisinger, T. Grasser, W. Gustin, and C. Schlünder, "The Statistical Analysis of Individual Defects Constituting NBTI and its Implications for Modeling DC- and AC-Stress," in Proc. Intl.Rel.Phys.Symp. (IRPS), May 2010, pp. 7-15.

[22] K. Zhao, J. Stathis, B. Linder, E. Cartier, and A. Kerber, "PBTI Under Dynamic Stress: From a Single Defect Point of View," in Proc. Intl.Rel.Phys.Symp. (IRPS), Apr. 2011, pp. 372-380.

[23] T. Grasser, P.-J. Wagner, H. Reisinger, T. Aichinger, G. Pobegen, M. Nelhiebel, and B. Kaczer, "Analytic Modeling of the Bias Temperature Instability Using Capture/Emission Time Maps," in Proc. Intl.Electron Devices Meeting (IEDM), Dec. 2011, pp. 27.4.1-27.4.4.

[24] T. Grasser, H. Reisinger, P.-J. Wagner, W. Goes, F. Schanovsky, and B. Kaczer, "The Time Dependent Defect Spectroscopy (TDDS) for the Characterization of the Bias Temperature Instability," in Proc. Intl.Rel.Phys.Symp. (IRPS), May 2010, pp. 16-25.

[25] T. Grasser, B. Kaczer, W. Goes, H. Reisinger, T. Aichinger, P. Hehenberger, P.-J. Wagner, F. Schanovsky, J. Franco, P. Roussel, and M. Nelhiebel, "Recent Advances in Understanding the Bias Temperature Instability," in Proc. Intl.Electron Devices Meeting (IEDM), Dec. 2010, pp. $82-85$.

[26] T. Tewksbury, "Relaxation Effects in MOS Devices due to Tunnel Exchange with Near-Interface Oxide Traps," Ph.D. Thesis, MIT, 1992.

[27] T. Grasser, "Stochastic Charge Trapping in Oxides: From Random Telegraph Noise to Bias Temperature Instabilities," in Microelectronics Reliability, vol. 52, 2012, pp. 39-70.

[28] V. Huard, M. Denais, and C. Parthasarathy, "NBTI Degradation: From Physical Mechanisms to Modelling," Microelectronics Reliability, vol. 46, no. 1, pp. 1-23, 2006.

[29] A. Islam, H. Kufluoglu, D. Varghese, S. Mahapatra, and M. Alam, "Recent Issues in Negative-Bias Temperature Instability: Initial Degradation, Field Dependence of Interface Trap Generation, Hole Trapping Effects, and Relaxation," IEEE Trans.Electron Devices, vol. 54, no. 9, pp. 21432154, 2007.

[30] M. Masuduzzaman, A. Islam, and M. Alam, "Exploring the Capability of Multifrequency Charge Pumping in Resolving Location and Energy Levels of Traps Within Dielectric," IEEE Trans.Electron Devices, vol. 55, no. 12, pp. 3421-3431, 2008.

[31] T. Grasser, B. Kaczer, W. Goes, T. Aichinger, P. Hehenberger, and M. Nelhiebel, "A Two-Stage Model for Negative Bias Temperature Instability," in Proc. Intl.Rel.Phys.Symp. (IRPS), 2009, pp. 33-44.

[32] M. Toledano-Luque, B. Kaczer, E. Simoen, P. Roussel, A. Veloso, T. Grasser, and G. Groeseneken, "Temperature and Voltage Dependences of the Capture and Emission Times of Individual Traps in High- $\kappa$ Dielectrics," in Proc. Insulating Films Semicond. (INFOS), 2011.

[33] M. Toledano-Luque, B. Kaczer, P. Roussel, T. Grasser, G. Wirth, J. Franco, C. Vrancken, N. Horiguchi, and G. Groeseneken, "Response of a single trap to AC Negative Bias Temperature Stress," in Proc. Intl.Rel.Phys.Symp. (IRPS), 2011, pp. 364-371.

[34] M. Toledano-Luque, B. Kaczer, E. Simoen, P. Roussel, A. Veloso, T. Grasser, and G. Groeseneken, "Temperature and Voltage Dependences of the Capture and Emission Times of Individual Traps in High- $\kappa$ Dielectrics," Microelectronic Engineering, vol. 88, pp. 1243-1246, 2011.

[35] A. Islam, S. Mahapatra, S. Deora, V. Maheta, and M. Alam, "Essential Aspects of Negative Bias Temperature Instability (NBTI)," http://www.nanohub.org, 2011

[36] B. Kaczer, V. Arkhipov, R. Degraeve, N. Collaert, G. Groeseneken, and M. Goodwin, "Disorder-Controlled-Kinetics Model for Negative Bias Temperature Instability and its Experimental Verification," in Proc. Intl.Rel.Phys.Symp. (IRPS), 2005, pp. 381-387.

[37] T. Grasser, B. Kaczer, and W. Goes, "An Energy-Level Perspective of Bias Temperature Instability," in Proc. Intl.Rel.Phys.Symp. (IRPS), 2008, pp. $28-38$.

[38] T. Aichinger, M. Nelhiebel, and T. Grasser, "Unambiguous Identification of the NBTI Recovery Mechanism using Ultra-Fast Temperature Changes," in Proc. Intl.Rel.Phys.Symp. (IRPS), 2009, pp. 2-7.

[39] T. Grasser, T. Aichinger, G. Pobegen, H. Reisinger, P.-J. Wagner, J. Franco, M. Nelhiebel, and B. Kaczer, "The 'Permanent' Component of NBTI: Composition and Annealing," in Proc. Intl.Rel.Phys.Symp. (IRPS), Apr. 2011, pp. 605-613.

[40] T. Grasser, H. Reisinger, P.-J. Wagner, and B. Kaczer, "The Time Dependent Defect Spectroscopy for the Characterization of Border Traps in Metal-Oxide-Semiconductor Transistors," Physical Review B, vol. 82, no. 24 , p. $245318,2010$. 